\title{
Endocrine immune-related adverse events: a double-edged sword?
}

\author{
Michelle Rengarajan ${ }^{1}$, Justin F. Gainor ${ }^{2}$ \\ ${ }^{1}$ Division of Endocrinology, Department of Medicine, Massachusetts General Hospital, Boston, MA, USA; ${ }^{2}$ Division of Hematology/Oncology, \\ Department of Medicine, Massachusetts General Hospital, Boston, MA, USA \\ Correspondence to: Justin F. Gainor, MD. Massachusetts General Hospital, 10 North Grove Street, Office 304, Boston, MA 02114, USA. \\ Email: jgainor@mgh.harvard.edu. \\ Comment on: España S, Pérez Montes de Oca A, Marques-Pamies M, et al. Endocrine adverse events related to immune-oncology agents: \\ retrospective experience of a single institution. Transl Lung Cancer Res 2020;9:103-10.
}

Submitted Jul 28, 2020. Accepted for publication Aug 21, 2020.

doi: 10.21037/tlcr-2020-16

View this article at: http://dx.doi.org/10.21037/tlcr-2020-16

Immune checkpoint inhibitors (ICIs) have transformed cancer care over the last decade. Currently, ICIs targeting the programmed cell death $1(\mathrm{PD}-1)$ axis are approved in more than 15 cancer types and are considered cornerstones of the management of advanced non-small cell lung cancer, melanoma and head and neck cancers, among others. Despite sometimes dramatic and durable responses, treatment with ICIs can be complicated by severe and even life-threatening immune related adverse events (IRAEs). These IRAEs occur when the immune system, activated by ICI treatment, attacks organ systems in a manner akin to organ-specific autoimmunity. IRAEs occur in many organs, including cardiovascular, pulmonary, GI, renal, musculoskeletal, dermatologic and endocrine systems (1). In severe cases, IRAEs may necessitate discontinuation of ICI treatment, which may worsen control of a patient's cancer; as such IRAEs have been referred to as the Achilles heel of immunotherapy (2).

In this issue of Translational Lung Cancer Research, España et al. perform a large retrospective analysis of endocrine IRAEs in a cohort of 260 patients with solid tumors, predominately NSCLC, who were treated with ICIs within a single institution. They find that $18 \%$ of patients develop one or more endocrine IRAEs, the majority of which are thyroiditis (82\% of endocrine IRAEs). Interestingly, 74\% of patients with thyroiditis in this series exhibited isolated subclinical or clinical hypothyroidism, without a preceding hyperthyroid phase. After correcting for differences in the frequency of use across ICI agents, endocrine IRAEs were most likely to develop in patients on combination ipilimumab/nivolumab (64\%), with lower rates observed among patients receiving PD-1 (16\%) or PD-L1 (20\%) inhibitor monotherapy, consistent with observations from earlier studies (3-5). Importantly, España and colleagues also suggest that the presence of an ICI-induced endocrinopathy was associated with improved overall and progression-free survival (OS: not calculable vs. 31.4 months and PFS: 56.7 vs. 27.7 months, respectively).

The findings by España and colleagues raise several important considerations. The first centers around the observed improvements in PFS and OS. A major challenge of dissecting the relationship between IRAEs and efficacy outcomes is the potential for guarantee-time bias (6). Guarantee-time bias occurs when an outcome is timed from the start of study enrollment and is compared across groups, but membership in a group is defined by an event that occurs anytime during follow-up. In this case, the outcome, PFS or OS, is timed from initiation of ICI therapy and is compared across patient groups, which are defined by whether or not patients develop IRAEs at any time after ICI initiation. Patients with favorable responses to ICIs may stay on treatment for longer; thus, they are more likely to have greater exposure to therapy (i.e., repeat administrations) and also have more time to develop IRAEs compared to patients who progressed through therapy and may not have survived long enough to develop IRAEs (2). España et al. suggest that IRAEs that occur early after initial exposure to a given therapy may be less subject to this bias, because the guarantee time, defined as the time to developing an IRAE, would be shorter relative to PFS or OS. In their cohort, endocrine IRAEs generally occurred early after ICI initiation—on average after 8 weeks. Nonetheless, it should 
be emphasized that a majority of patients in this series had NSCLC and received PD-(L)1 inhibitors in the secondline setting. In randomized studies, median PFS on PD(L)1 inhibitors in previously-treated patients has been only 2-4 months (7). Thus, despite the relatively early-onset of endocrine IRAEs in the series by España and colleagues, guarantee-time bias likely still played a role in the above observations.

Recently, several other groups have also tried to perform early landmark analyses to tease apart relationships between the presence of IRAEs and efficacy of ICIs. A retrospective 6-week landmark analysis of patients with advanced and recurrent NSCLC, including only IRAEs occurring prior to the landmark date, found that patients with IRAEs exhibited prolonged PFS (9.2 vs. 4.8 months) and OS (not reached $v s .11 .1$ months) relative to patients without IRAEs (8). Similar prolongation of PFS and OS, along with improved disease control, was found in a prospective cohort study in which patients with advanced NSCLC were evaluated for IRAEs between the initiation of ICI and the first response evaluation (9). As more data has accumulated on ICI-thyroiditis, it appears to typically present within an early time window $(3,4,10)$ and has been associated with prolonged survival $(3,5)$ in prior studies as well. It is possible that early ICI-thyroiditis may be a marker of systemic immunomodulatory effects that are correlated with improved tumoral control; additional well-controlled studies may help to clarify this relationship.

The potential relationship between endocrine IRAEs and checkpoint inhibitor efficacy raises the possibility of shared immune mechanisms driving tumoral control and autoimmunity. Several mechanisms have been proposed that might explain this relationship, including both antigendependent mechanisms and mechanisms involving a broader increase in T-cell activity (2). Antigen-dependent mechanisms might link specific solid tumors to specific IRAEs. For instance, the development of vitiligo after ICI treatment is associated with prolonged survival in patients with melanoma $(11,12)$. Since melanoma cells and normal melanocytes share common surface antigens $(13,14)$, ICI treatment may cause proliferation of T-cells that target these shared antigens and contribute to both vitiligo and tumor response. In contrast, no clear relationship between endocrine IRAEs and particular tumor types has yet been reported. Instead, it is possible that endocrine IRAEs are a marker of decreased T-cell activation thresholds or increased reinvigoration of exhausted T-cells. Both IRAEs and improved tumoral control may result from increased activation of both self-reactive and tumor-reactive T-cells, respectively. Mechanistic studies of immune cells from patients treated with ICI with and without IRAEs are necessary to elucidate cellular phenotypes that drive this possible link between ICI efficacy and IRAEs.

One further challenge in deciphering the relationship between endocrine IRAEs and tumoral control is that in most studies, endocrine IRAEs are considered collectively; however, the clinical features of each IRAE can be quite distinct. For instance, ICI-hypophysitis occurs frequently (11-13\%) after treatment with the CTLA4 inhibitor ipilimumab, but is rare $(0.5 \%)$ following treatment with PD1 inhibitors $(15,16)$. Data from mouse models suggests that this bias with ipilimumab is a direct consequence of CTLA4 expression by hormone-producing pituitary cells with glandular destruction resulting from antibodydependent, cell-mediated cytotoxicity (17). In contrast, ICI-thyroiditis may be more common after treatment with PD1 inhibitors (18) relative to ipilimumab; this may relate to PD-L1 expression in the thyroid $(19,20)$. Likewise, the timing of onset of distinct endocrinopathies can vary significantly: from an average of 37 days after ICI initiation for thyroiditis (10), 65 days after initiation of ipilimumab for hypophysitis (15), to 116 days after ICI initiation for diabetes (21). Thus, whereas it is tempting to speculate that a unifying mechanism drives immune-mediated destruction of hormone-producing cells, in the absence of clear evidence for such a shared mechanism, we favor considering distinct endocrine IRAEs as distinct entities.

Clinically, one challenge in identifying endocrine IRAEs is that the diagnostic tests used to define these entitiessuch as thyroid stimulating hormone (TSH), free thyroxine (free T4), cortisol, adrenocorticotropic hormone (ACTH), blood glucose, C-peptide- - tend to be markers of glandular function rather than glandular integrity. For instance, a patient treated with a PD1 inhibitor may have immune infiltration of the thyroid gland, but ICI-thyroiditis is generally not diagnosed clinically until the level of immunemediated destruction is sufficient to alter thyroid function by changing TSH or free T4 levels. This contrasts with non-endocrine IRAEs such as hepatitis, for which AST and ALT may reflect organ damage independent of function. Glandular function is the output of a balance between immune-mediated glandular destruction as well as glandular regeneration. Thus, it is possible that the ICIdriven destructive process differs across endocrinopathies. Alternatively, clinical differences may reflect differences in regenerative capacity of the pituitary, pancreas, and thyroid. 
Additionally, many cases of "subclinical" endocrine IRAEs, in which patients have immune-mediated glandular damage but retain normal glandular function, may be missed. While these cases may not have significant clinical consequences, they may be important to understand the underlying basis of endocrine IRAEs.

Notably, for ICI-thyroiditis in particular, a number of different functional phenotypes have been reported: (I) the classic pattern of thyroiditis, with an initial thyrotoxic phase transitioning to hypothyroidism, (II) self-limited thyrotoxicosis, (III) isolated hypothyroidism, (IV) subclinical thyrotoxicosis, and (V) subclinical hypothyroidism. Presumably, these represent a spectrum of a single disease entity with a shared underlying mechanism, but this has not yet been shown. Because the thyrotoxicosis associated with ICI thyroiditis is attributed to damageinduced release of preformed hormone, it is possible that the presence of a thyrotoxic phase reflects more rapidly aggressive glandular destruction, particularly in patients with the classic pattern. Clinically, dual checkpoint blockade (e.g., PD-1 plus CTLA-4 inhibition) is associated with more IRAEs than monotherapy, perhaps due to broader and more robust reinvigoration of the immune system. Notably, a recently published meta-analysis suggested that in patients treated with combination nivolumab plus ipilimumab, almost all patients with ICI thyroiditis (93.1\%) had the classic presentation (1), compared to a smaller percentage of patients with ICI thyroiditis after nivolumab or pembrolizumab monotherapy $(63.3 \%$ and $73.5 \%$, respectively) (22).

Thyroid-specific autoantibodies are another marker of autoimmunity and offer an opportunity to further probe the mechanistic basis of ICI thyroiditis as well as differences among functional phenotypes. Interestingly, España $e t$ al. report positive antithyroid antibodies in $5 / 7$ patients who present with the classic pattern, though the details of whether these antibodies target thyroid peroxidase (TPO) or thyroglobulin $(\mathrm{Tg})$ are elided, as is the prevalence of these antithyroid antibodies in patients with other functional phenotypes of ICI thyroiditis. Anti-TPO and anti-Tg antibodies are strongly associated with a spontaneous autoimmune thyroiditis, Hashimoto's thyroiditis; in particular, $>90 \%$ of patients with Hashimoto's thyroiditis have elevated anti-TPO antibodies. A high prevalence of anti-TPO and anti-Tg antibodies in patients with ICI thyroiditis might suggest a mechanistic link between IRAEs (ICI-thyroiditis) and spontaneous autoimmune disease
(Hashimoto's thyroiditis). In the general population, antiTPO and anti-Tg antibodies are present in approximately $11-13 \%$ of individuals without thyroid dysfunction $(23,24)$. These antibodies are associated with immune infiltration of the thyroid gland (25), and these individuals are at increased risk of developing hypothyroidism (26). Thus, anti-TPO and anti-Tg antibodies may be markers of glandular integrity that are independent of glandular function.

If anti-TPO and anti-Tg antibodies predict ICI thyroiditis, they might prospectively identify patients at high risk of developing ICI thyroiditis and might also suggest a shared immune mechanism with Hashimoto's thyroiditis. When anti-TPO and anti-Tg antibodies were prospectively followed in patients enrolled at one institution in the KEYNOTE-001 trial, 8\% (4/48) of patients with normal thyroid function had anti-TPO or anti-Tg antibodies prior to the start of immunotherapy, and 3 of these 4 patients developed ICI thyroiditis; in comparison, only $16 \%$ of patients without antibodies at the start of therapy developed ICI thyroiditis (5). During the course of the KEYNOTE-001 trial, another 7 patients developed autoantibodies; including these patients, $80 \%$ of patients with ICI thyroiditis had positive autoantibodies, compared with only $8 \%$ of patients without ICI thyroiditis (5). Collectively, these observations suggest that anti-TPO and anti-Tg antibodies are enriched among patients who develop ICI thyroiditis, suggesting a possible link to Hashimoto's thyroiditis. Furthermore, while far more data is necessary, the increased prevalence of ICI-thyroiditis among patients with pre-existing anti-TPO and anti-Tg antibodies suggest that ICIs may drive pre-existing immune infiltration of the thyroid gland toward a functional deficit.

In summary, endocrine IRAEs are a common complication of ICI treatment, and close monitoring of endocrine function should accompany ICI administration to ensure rapid diagnosis and treatment if these IRAEs develop. Despite the frequency of these events, the mechanism underlying endocrine IRAEs remains unclear and likely involves a complex interplay of T-cell activation and glandular regeneration that may be related to spontaneous organ-specific autoimmunity. Given the intriguing possibility that IRAEs are correlated with improved disease outcomes, PFS and OS, understanding the mechanistic basis of endocrine IRAEs may also reveal insights into antitumor efficacy of ICIs and facilitate further optimization of immunomodulatory therapy in cancer. 


\section{Acknowledgments}

Funding: None.

\section{Footnote}

Provenance and Peer Review: This article was commissioned by the editorial office, Translational Lung Cancer Research. The article did not undergo external peer review.

Conflicts of Interest: Both authors have completed the ICMJE uniform disclosure form (available at http://dx.doi. org/10.21037/tlcr-2020-16). MR reports grants from NIH T32 DK007028, outside the submitted work. JFG reports grants and personal fees from Bristol-Myers Squibb, grants and personal fees from Genentech/Roche, personal fees from Takeda, grants and personal fees from Blueprint, grants and personal fees from Loxo, personal fees from Oncorus, personal fees from Regeneron, personal fees from Pfizer, personal fees from Incyte, grants and personal fees from Novartis, grants and personal fees from Merck, personal fees from Agios, personal fees from Amgen, grants from Array, grants from Tesaro, grants from Moderna, grants from Adaptimmune, grants from Alexo, personal fees from Ironwood Pharmaceuticals, personal fees from Gilead, outside the submitted work.

Ethical Statement: The authors are accountable for all aspects of the work in ensuring that questions related to the accuracy or integrity of any part of the work are appropriately investigated and resolved.

Open Access Statement: This is an Open Access article distributed in accordance with the Creative Commons Attribution-NonCommercial-NoDerivs 4.0 International License (CC BY-NC-ND 4.0), which permits the noncommercial replication and distribution of the article with the strict proviso that no changes or edits are made and the original work is properly cited (including links to both the formal publication through the relevant DOI and the license). See: https://creativecommons.org/licenses/by-nc-nd/4.0/.

\section{References}

1. Postow MA, Sidlow R, Hellmann MD. Immune-Related Adverse Events Associated with Immune Checkpoint Blockade. N Engl J Med 2018;378:158-68.

2. June CH, Warshauer JT, Bluestone JA. Corrigendum: Is autoimmunity the Achilles' heel of cancer immunotherapy? Nat Med 2017;23:1004.

3. Kotwal A, Kottschade L, Ryder M. PD-L1 InhibitorInduced Thyroiditis Is Associated with Better Overall Survival in Cancer Patients. Thyroid 2020;30:177-84.

4. Delivanis DA, Gustafson MP, Bornschlegl S, et al. Pembrolizumab-Induced Thyroiditis: Comprehensive Clinical Review and Insights Into Underlying Involved Mechanisms. J Clin Endocrinol Metab 2017;102:2770-80.

5. Osorio JC, Ni A, Chaft JE, et al. Antibody-mediated thyroid dysfunction during T-cell checkpoint blockade in patients with non-small-cell lung cancer. Ann Oncol 2017;28:583-9.

6. Giobbie-Hurder A, Gelber RD, Regan MM. Challenges of guarantee-time bias. J Clin Oncol 2013;31:2963-9.

7. Herzberg B, Campo MJ, Gainor JF. Immune Checkpoint Inhibitors in Non-Small Cell Lung Cancer. Oncologist 2017;22:81-8

8. Haratani K, Hayashi H, Chiba Y, et al. Association of Immune-Related Adverse Events With Nivolumab Efficacy in Non-Small-Cell Lung Cancer. JAMA Oncol 2018;4:374-8.

9. Teraoka S, Fujimoto D, Morimoto T, et al. Early ImmuneRelated Adverse Events and Association with Outcome in Advanced Non-Small Cell Lung Cancer Patients Treated with Nivolumab: A Prospective Cohort Study. J Thorac Oncol 2017;12:1798-805.

10. Iyer PC, Cabanillas ME, Waguespack SG, et al. ImmuneRelated Thyroiditis with Immune Checkpoint Inhibitors. Thyroid 2018;28:1243-51.

11. Lo JA, Fisher DE, Flaherty KT. Prognostic Significance of Cutaneous Adverse Events Associated With Pembrolizumab Therapy. JAMA Oncol 2015;1:1340-1.

12. Hua C, Boussemart L, Mateus C, et al. Association of Vitiligo With Tumor Response in Patients With Metastatic Melanoma Treated With Pembrolizumab. JAMA Dermatol 2016;152:45-51.

13. Houghton AN, Eisinger M, Albino AP, et al. Surface antigens of melanocytes and melanomas. Markers of melanocyte differentiation and melanoma subsets. J Exp Med 1982;156:1755-66.

14. Cui J, Bystryn JC. Melanoma and vitiligo are associated with antibody responses to similar antigens on pigment cells. Arch Dermatol 1995;131:314-8.

15. Faje A, Reynolds K, Zubiri L, et al. Hypophysitis secondary to nivolumab and pembrolizumab is a clinical entity distinct from ipilimumab-associated hypophysitis. Eur J Endocrinol 2019;181:211-9. 
16. Faje AT, Sullivan R, Lawrence D, et al. Ipilimumabinduced hypophysitis: a detailed longitudinal analysis in a large cohort of patients with metastatic melanoma. J Clin Endocrinol Metab 2014;99:4078-85.

17. Iwama S, De Remigis A, Callahan MK, et al. Pituitary expression of CTLA-4 mediates hypophysitis secondary to administration of CTLA-4 blocking antibody. Sci Transl Med 2014;6:230ra45.

18. Barroso-Sousa R, Barry WT, Garrido-Castro AC, et al. Incidence of Endocrine Dysfunction Following the Use of Different Immune Checkpoint Inhibitor Regimens: A Systematic Review and Meta-analysis. JAMA Oncol 2018;4:173-82.

19. Neppl C, Kaderli RM, Trepp R, et al. Histology of Nivolumab-Induced Thyroiditis. Thyroid 2018;28:1727-8.

20. Imblum BA, Baloch ZW, Fraker D, et al. PembrolizumabInduced Thyroiditis. Endocr Pathol 2019;30:163-7.

21. Wright JJ, Salem JE, Johnson DB, et al. Increased Reporting of Immune Checkpoint Inhibitor-Associated

Cite this article as: Rengarajan M, Gainor JF. Endocrine immune-related adverse events: a double-edged sword? Transl Lung Cancer Res 2021;10(1):13-17. doi: 10.21037/tlcr-2020-16
Diabetes. Diabetes Care 2018;41:e150-1.

22. Muir CA, Menzies AM, Clifton-Bligh R, et al. Thyroid Toxicity Following Immune Checkpoint Inhibitor Treatment in Advanced Cancer. Thyroid 2020;30:1458-69

23. Hackett E, Beech M, Forbes IJ. Thyrogloubulin antibodies in patients without clinical disease of the thyroid gland. Lancet 1960;2:402-4.

24. Hollowell JG, Staehling NW, Flanders WD, et al. Serum TSH, T(4), and thyroid antibodies in the United States population (1988 to 1994): National Health and Nutrition Examination Survey (NHANES III). J Clin Endocrinol Metab 2002;87:489-99.

25. Yoshida H, Amino N, Yagawa K, et al. Association of serum antithyroid antibodies with lymphocytic infiltration of the thyroid gland: studies of seventy autopsied cases. J Clin Endocrinol Metab 1978;46:859-62.

26. Dayan CM, Daniels GH. Chronic autoimmune thyroiditis. N Engl J Med 1996;335:99-107. 